\title{
ON THE EQUATION SATISFIED BY A STEADY PRANDTL-MUNK VORTEX SHEET*
}

\author{
MILTON C. LOPES FILHO ${ }^{\dagger}$, HELENA J. NUSSENZVEIG LOPES ${ }^{\ddagger}$, AND MAX O. SOUZA
}

\begin{abstract}
We show that the vorticity distribution obtained by minimizing the induced drag on a wing, the so called Prandtl-Munk vortex sheet, is not a travelling-wave weak solution of the Euler equations, contrary to what has been claimed by a number of authors. Instead, it is a weak solution of a non-homogeneous Euler equation, where the forcing term represents a "tension" force applied to the tips. This is consistent with a heuristic argument due to Saffman. Thus, the notion of weak solution captures the correct physical behavior in this case.
\end{abstract}

The Prandtl-Munk vortex sheet is notorious in fluid dynamics as the one that generates a circulation distribution that minimizes the induced drag on a plane wing $[1,8]$. The vorticity associated to the Prandtl-Munk vortex sheet is given by

$$
\omega_{0}\left(x_{1}, x_{2}\right)=\frac{x_{1}}{\sqrt{1-x_{1}^{2}}} \chi_{(-1,1)}\left(x_{1}\right) \otimes \delta_{0}\left(x_{2}\right),
$$

where $\chi_{(-1,1)}$ represents the characteristic function of the interval $(-1,1)$ and $\delta_{0}$ is the Dirac delta at $x_{2}=0$.

The associated velocity, given by the Biot-Savart law, can be evaluated at points on the sheet by means of a principal value integral and is found to be constant and equal to $(0,-1 / 2)$ [8]. Hence a natural candidate for a weak solution is determined by the steadily translating vorticity profile:

$$
\omega\left(x_{1}, x_{2}, t\right)=\omega_{0}\left(x_{1}, x_{2}+\frac{t}{2}\right) .
$$

A number of authors $[3,6]$ have claimed, albeit with some doubts, that (2) is indeed a weak solution of the steady Euler equations. However, the numerical experiments performed by Krasny [3] suggest that the (physically correct) solution of the Euler equation with (1) as initial data is not steady, actually rolling-up at the tips for positive time. Later, Saffman [8] gave a heuristic argument, based on vortex principles, as to why the steady solution (2) is not dynamically consistent. This scenario suggests that the Prandtl-Munk vortex profile (1) is an example of nonuniqueness for the weak formulation of vortex sheet evolution. We shall show that (2) is actually a weak solution of a nonhomogeneous Euler equation, with forcing term consistent with that obtained by Saffman, thus ruling out the Prandtl-Munk vortex sheet as an example of nonuniqueness.

It is not known to which degree unphysical flows may satisfy the standard notion of weak solution to the Euler equations. Examples of (unphysical) weak solutions with kinetic energy growing in time have been obtained $[9,11]$ in a situation considerably more irregular than vortex sheet dynamics. The main point of the present

\footnotetext{
*Received: April 15, 2002; Accepted (in revised version): April 25, 2002.

${ }^{\dagger}$ Departamento de Matematica, IMECC-UNICAMP. Caixa Postal 6065, Campinas, SP 13081-970, Brasil (mlopes@ime.unicamp.br).

${ }^{\ddagger}$ Departamento de Matematica, IMECC-UNICAMP. Caixa Postal 6065, Campinas, SP 13081-970, Brasil (hlopes@ime.unicamp.br).

$\S$ Departamento de Matematica, PUC-Rio, Rua Marquês de São Vicente, 225 Rio de Janeiro RJ, 22453-900, Brasil (msouza@mat.puc-rio.br). Present address: Depto. de Matematica Aplicada, UFF, Rua Mario Santos Braga s/n, Niteroi, RJ 24020-140, Brasil.
} 
paper is the fact that the notion of weak solution is discriminating enough to distinguish the physically correct evolution for the Prandtl-Munk vortex sheet, something which cannot be done through the Birkhoff-Rott model alone. A different example of nonphysical weak solution in the context of vortex sheets has been proposed and numerically studied, see Lopes Filho et al. [4].

The standard definition of a weak solution of the Euler equations uses velocity as the main dynamic variable [5]. We observe that it is not easy to check, using this notion of weak solution, whether (2) is or is not a weak solution of the Euler equations. Indeed, despite the fact that the vorticity associated to the Prandtl-Munk vortex sheet has the particularly simple form (1), the associated velocity is fairly complicated [8]. For this reason, we choose to work with the vorticity equation, and we will use the weak vorticity formulation originally introduced by Delort [2] and reformulated by Schochet [10] stated as follows: a measure $\omega \in L^{\infty}\left(\left[(0, \infty) ; \mathcal{B M}\left(\mathbb{R}^{2}\right) \cap H_{\text {loc }}^{-1}\left(\mathbb{R}^{2}\right)\right)\right.$ is a weak solution of the inhomogeneous vorticity formulation of the incompressible 2D Euler equations with initial data $\omega_{0} \in \mathcal{B M}_{c}\left(\mathbb{R}^{2}\right) \cap H^{-1}\left(\mathbb{R}^{2}\right)$, and forcing $F \in$ $L^{\infty}\left(\left[(0, \infty) ; W_{\text {loc }}^{-1,1}\left(\mathbb{R}^{2}\right)\right)\right.$ if, for any test function $\varphi \in C_{c}^{\infty}\left(\left[(0, \infty) \times \mathbb{R}^{2}\right)\right.$, we have:

$$
\begin{array}{r}
\int_{0}^{\infty}\left(\int_{\mathbb{R}^{2}} \varphi_{t} \mathrm{~d} \omega(x, t)+\int_{\mathbb{R}^{2}} \int_{\mathbb{R}^{2}} H_{\varphi} d \omega(x, t) \otimes \mathrm{d} \omega(y, t)\right) \mathrm{d} t+ \\
\int_{R^{2}} \varphi(x, 0) \mathrm{d} \omega_{0}(x)=\int_{0}^{\infty} \int_{\mathbb{R}^{2}} \nabla^{\perp} \varphi \cdot \mathrm{d} F(x, t) \mathrm{d} t,
\end{array}
$$

where

$$
H_{\varphi}(x, y, t) \equiv \frac{\nabla \varphi(x, t)-\nabla \varphi(y, t)}{4 \pi|x-y|} \cdot \frac{(x-y)^{\perp}}{|x-y|} .
$$

In the homogeneous case, this formulation was shown to be equivalent to the standard velocity one, as long as the velocity is $\left(L_{\text {loc }}^{2}\left(\mathbb{R}^{2}\right)\right)^{2}$, by Delort [2]. However, the proof carries over in a straightforward manner to the inhomogeneous case.

Using the formulation above, we shall show that the velocity associated to (2) solves, in a weak sense, the system of equations:

$$
\left\{\begin{array}{l}
u_{t}+u \cdot \nabla u=-\nabla p+F \\
\operatorname{div} u=0
\end{array}\right.
$$

with the forcing term

$$
F\left(x_{1}, x_{2}\right)=-\pi / 8\left[\begin{array}{c}
\delta_{0}\left(x_{1}-1, x_{2}+t / 2\right)-\delta_{0}\left(x_{1}+1, x_{2}+t / 2\right) \\
0
\end{array}\right] .
$$

The factor in (4) should be compared with equation (7) in $\S 6.3$ of Saffman [8], keeping in mind that, in our case, we have $U=1 / 2$ and $a=1$. Thus, in accordance with Saffman's analysis, the forcing given by (4) can be thought of as a tension applied near the tips of the vortex sheet.

We begin by choosing a test function of the form

$$
\varphi\left(x_{1}, x_{2}, t\right)=\alpha(t) \zeta\left(x_{1}, x_{2}+\frac{t}{2}\right)
$$

where $\alpha$ and $\zeta$ are $C_{c}^{\infty}$ in $[0, \infty)$ and $\mathbb{R}^{2}$, respectively. We notice that finite sums of test functions having this form are dense in the set of all $C_{c}^{\infty}\left([0, \infty) \times \mathbb{R}^{2}\right)$ test functions.

We now compute the right-hand side of (3) with $\omega$ given by (2): 
70 ON THE EQUATION SATISFIED BY A STEADY PRANDTL-MUNK VORTEX SHEET

(i)

$$
\begin{gathered}
\int_{0}^{\infty} \int_{\mathbb{R}^{2}} \varphi_{t} \mathrm{~d} \omega(x, t) \mathrm{d} t= \\
\int_{0}^{\infty} \int_{-1}^{1}\left(\alpha^{\prime}(t) \zeta\left(x_{1}, 0\right)+\alpha(t) \frac{1}{2} \zeta_{x_{2}}\left(x_{1}, 0\right)\right) \frac{x_{1}}{\sqrt{1-x_{1}^{2}}} \mathrm{~d} x_{1} \mathrm{~d} t= \\
-\int_{-1}^{1} \alpha(0) \zeta\left(x_{1}, 0\right) \frac{x_{1}}{\sqrt{1-x_{1}^{2}}} d x_{1}+\int_{0}^{\infty} \alpha(t) \frac{1}{2} \int_{-1}^{1} \zeta_{x_{2}}\left(x_{1}, 0\right) \frac{x_{1}}{\sqrt{1-x_{1}^{2}}} \mathrm{~d} x_{1} \mathrm{~d} t
\end{gathered}
$$

(ii)

$$
\begin{gathered}
\int_{0}^{\infty} \int_{\mathbb{R}^{2}} \int_{\mathbb{R}^{2}} H_{\varphi} \mathrm{d} \omega(x, t) \otimes d \omega(y, t) \mathrm{d} t= \\
\int_{0}^{\infty} \alpha(t) \frac{1}{4 \pi} \int_{-1}^{1} \int_{-1}^{1} \frac{\zeta_{x_{2}}\left(x_{1}, 0\right)-\zeta_{x_{2}}\left(y_{1}, 0\right)}{x_{1}-y_{1}} \frac{x_{1}}{\sqrt{1-x_{1}^{2}}} \frac{y_{1}}{\sqrt{1-y_{1}^{2}}} \mathrm{~d} x_{1} \mathrm{~d} y_{1} \mathrm{~d} t
\end{gathered}
$$

(iii)

$$
\int_{R^{2}} \varphi(x, 0) \mathrm{d} \omega_{0}(x)=\int_{-1}^{1} \alpha(0) \zeta\left(x_{1}, 0\right) \frac{x_{1}}{\sqrt{1-x_{1}^{2}}} \mathrm{~d} x_{1} .
$$

adding the three terms, dropping the subscript in $x_{1}$ and in $y_{1}$, and writing $\zeta_{x_{2}}=\eta(x)$, we obtain:

$$
\begin{gathered}
\int_{0}^{\infty}\left(\int_{\mathbb{R}^{2}} \varphi_{t} \mathrm{~d} \omega(x, t)+\int_{\mathbb{R}^{2}} \int_{\mathbb{R}^{2}} H_{\varphi} \mathrm{d} \omega(x, t) \otimes \mathrm{d} \omega(y, t)\right) \mathrm{d} t+\int_{R^{2}} \varphi(x, 0) \mathrm{d} \omega_{0}(x)= \\
\frac{1}{2} \int_{-1}^{1} \eta(x) \frac{x}{\sqrt{1-x^{2}}} \mathrm{~d} x+\frac{1}{4 \pi} \int_{-1}^{1} \int_{-1}^{1} \frac{\eta(x)-\eta(y)}{x-y} \frac{x}{\sqrt{1-x^{2}}} \frac{y}{\sqrt{1-y^{2}}} \mathrm{~d} x \mathrm{~d} y=\langle S, \eta\rangle .
\end{gathered}
$$

We now show that

$$
S=\frac{\pi}{8}\left(\delta_{1}-\delta_{-1}\right)
$$

We start by observing that the support of $S$ is obviously contained in the closed interval $[-1,1]$.

Next we observe that $S$ is an odd distribution, that is, if $\eta$ is an even test function then $\langle S, \eta\rangle=0$. Indeed, the first integral vanishes trivially, whereas to see that the second vanishes it suffices to use the change of variables $(x, y) \mapsto(-x,-y)$.

Now we show that $\langle S, \eta\rangle$ vanishes for any $\eta$ which is a polynomial vanishing at $x=1$ and $x=-1$ simultaneously. Clearly it is enough to verify this for all test functions of the form $\eta_{n}(x)=\left(1-x^{2}\right) x^{2 n-1}, n=1,2, \ldots$.

Observe that, for all $k=1,2, \ldots$, we have that

$$
\int_{-1}^{1} \frac{x^{2 k}}{\sqrt{1-x^{2}}} \mathrm{~d} x=\int_{0}^{\pi}(\cos \theta)^{2 k} \mathrm{~d} \theta=\frac{(2 k-1) ! !}{(2 k) ! !} \pi,
$$


where

$$
(2 n) ! !=\prod_{\ell=1}^{n}(2 \ell),(2 n-1) ! !=\prod_{\ell=1}^{n}(2 \ell-1)
$$

and the second equality is a well-known calculus identity [7].

Hence, the first term in (5) can be evaluated as

$$
\int_{-1}^{1} \eta_{n}(x) \frac{x}{\sqrt{1-x^{2}}} \mathrm{~d} x=\pi\left(\frac{(2 n-1) ! !}{(2 n) ! !}-\frac{(2 n+1) ! !}{(2 n+2) ! !}\right) .
$$

Since

$$
\frac{\eta_{n}(x)-\eta_{n}(y)}{x-y}=\sum_{\ell=0}^{2 n-2} x^{\ell} y^{2 n-2-\ell}-\sum_{\ell=0}^{2 n} x^{\ell} y^{2 n-\ell} ;
$$

the double integral in (5) can be evaluated, after noticing that even powers integrate to zero, as follows:

$$
\begin{gathered}
\int_{-1}^{1} \int_{-1}^{1} \frac{\eta_{n}(x)-\eta_{n}(y)}{x-y} \frac{x}{\sqrt{1-x^{2}}} \frac{y}{\sqrt{1-y^{2}}} \mathrm{~d} x \mathrm{~d} y= \\
\sum_{\ell=1}^{n-1} \int_{-1}^{1} \int_{-1}^{1} x^{2 \ell-1} y^{2 n-1-2 \ell} \frac{x}{\sqrt{1-x^{2}}} \frac{y}{\sqrt{1-y^{2}}} \mathrm{~d} x \mathrm{~d} y+ \\
-\sum_{\ell=1}^{n} \int_{-1}^{1} \int_{-1}^{1} x^{2 \ell-1} y^{2 n+1-2 \ell} \frac{x}{\sqrt{1-x^{2}}} \frac{y}{\sqrt{1-y^{2}}} \mathrm{~d} x \mathrm{~d} y= \\
\pi^{2}\left[\sum_{\ell=1}^{n-1} \frac{(2 \ell-1) ! !}{(2 \ell) ! !} \frac{(2 n-2 \ell-1) ! !}{(2 n-2 \ell) ! !}-\sum_{\ell=1}^{n} \frac{(2 \ell-1) ! !}{(2 \ell) ! !} \frac{(2 n-2 \ell+1) ! !}{(2 n-2 \ell+2) ! !}\right] .
\end{gathered}
$$

Thus, we want to show that

$$
\begin{array}{r}
0=\left\langle S, \eta_{n}\right\rangle=\frac{1}{2} \pi\left(\frac{(2 n-1) ! !}{(2 n) ! !}-\frac{(2 n+1) ! !}{(2 n+2) ! !}\right)+\frac{1}{4 \pi} \pi^{2} \\
{\left[\sum_{\ell=1}^{n-1} \frac{(2 \ell-1) ! !}{(2 \ell) ! !} \frac{(2 n-2 \ell-1) ! !}{(2 n-2 \ell) ! !}-\sum_{\ell=1}^{n} \frac{(2 \ell-1) ! !}{(2 \ell) ! !} \frac{(2 n-2 \ell+1) ! !}{(2 n-2 \ell+2) ! !}\right] .}
\end{array}
$$

Set

$$
a_{n}=\frac{(2 n+1) ! !}{(2 n+2) ! !}+\frac{1}{2} \sum_{\ell=1}^{n} \frac{(2 n+1-2 \ell) ! !}{(2 n+2-2 \ell) ! !} \frac{(2 \ell-1) ! !}{(2 \ell) ! !}
$$

for $n \geq 2$, and set $a_{0}=a_{1}=1 / 2$. With these definitions, (6) is equivalent to $a_{n-1}-a_{n}=0$.

Indeed, we now show that, for all $n=2,3, \ldots, a_{n}=1 / 2$. 
Write:

$$
b_{n}=\frac{(2 n+1) ! !}{(2 n+2) ! !}
$$

Then, with this notation we have:

$$
a_{n}=b_{n}+\frac{1}{2} \sum_{\ell=1}^{n} b_{n-\ell} b_{\ell-1}
$$

so that what we need to show is that the right-hand side of the equality above is identically equal to $1 / 2$, for $n=2,3, \ldots$. We will use a generating function argument.

We introduce the function:

$$
f(x)=\frac{1}{x}\left(\frac{1}{\sqrt{1-x}}-1\right) .
$$

It can be easily checked that the Taylor expansion of $f$ is

$$
f(x)=\sum_{n=0}^{\infty} b_{n} x^{n}
$$

Therefore, the identity sought reduces, through term-by-term identification of the Taylor coefficients, to the following algebraic identity:

$$
f(x)-\frac{1}{2}-\frac{3 x}{8}+\frac{x}{2}\left((f(x))^{2}-\frac{1}{4}\right)=\frac{1}{2}\left(\frac{1}{1-x}-1-x\right) .
$$

Since the $\eta_{n}$ are dense in $C_{c}^{\infty}((-1,1)), S$ vanishes against any such test functions. Thus, the support of $S$ is contained in $\{-1,1\}$ and, in this case, $S$ must be of the form

$$
S=\sum_{k=0}^{N} \alpha_{k}\left(\delta_{1}^{(k)}-(-1)^{k} \delta_{-1}^{(k)}\right)
$$

where $\alpha_{k} \in \mathbb{R}$, and we have taken advantage of the fact that $S$ is odd. Applying $S$ to $p(x)=x\left(1-x^{2}\right)^{n}$, we see that $\alpha_{n}=0, n \geq 1$. Finally, taking $\eta(x)=x$ shows that $\alpha_{0}=\pi / 8$.

Reverting back to our original notation, we have that (3) yields

$$
\int_{0}^{\infty} \alpha(t)\left(\frac{\pi}{8} \zeta_{x_{2}}(1,0)-\frac{\pi}{8} \zeta_{x_{2}}(-1,0)\right) \mathrm{d} t=\int_{0}^{\infty} \int_{\mathbb{R}^{2}} \nabla^{\perp} \varphi \cdot \mathrm{d} F(x, t) \mathrm{d} t,
$$

as claimed.

Acknowledgements. The authors would like to thank Robert Krasny and Oscar Orellana for their helpful comments. Part of this work was done during a visit of the third author to the first two, who thanks UNICAMP for the hospitality during the visit. M.C.L.F.'s research is supported in part by CNPq grant \# 300.962/91-6. H.J.N.L.'s research is supported in part by CNPq grant \# 300.158/93-9. This work was also supported by FAPESP grant \# 97/13855-0 and by PRONEX. 


\section{REFERENCES}

[1] G.K. Batchelor, An Introduction to Fluid Dynamics. Cambridge University Press, 1967.

[2] J.-M. Delort, Existence de nappes de tourbillon en dimension deux. J. of Amer. Math. Soc., 4:553-586, 1991.

[3] R. Krasny, Computation of vortex sheet roll-up in Trefftz plane. J. Fluid Mech., 184:123-155, 1987.

[4] M.C. Lopes Filho, J. Lowengrub, H.J. Nussenzveig Lopes, and Y. Zheng, Numerical evidence for the nonunique evolution of vortex sheets in the plane. Relatório de Pesquisa 44/99, IMECC-UNICAMP, 1999.

[5] C. Marchioro and M. Pulvirenti, Mathematical Theory of Incompressible Nonviscous Fluids. Springer-Verlag, 1994.

[6] R.E. Meyer, Introduction to Mathematical Fluid Dynamics. Dover, 1982.

[7] I.M. Ryzhik and I.S. Gradshteyn, Table of Integrals, Series and Products. Academic Press, 1994.

[8] P.G. Saffman, Vortex Dynamics. Cambridge University Press, 1992.

[9] V. Scheffer, An inviscid flow with compact support in space-time. J. of Geom. Anal., 3:343-401, 1993.

[10] S. Schochet, The weak vorticity formulation of the $2 d$ Euler equations and concentrationcancellation. Comm P.D.E., 20:1077-1104, 1995.

[11] A. Shnirelman, On the nonuniqueness of weak solutions of the Euler equations. Comm. Pure Appl. Math., 50:1261-1286, 1997. 